\title{
Determination of Endogenous Radioresistance Biomarkers on HeLa Cells
}

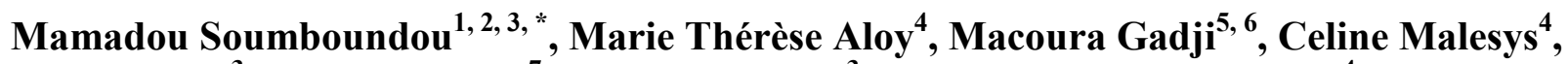 \\ Gora Mbaye $^{3}$, Ahmadou Dem ${ }^{7}$, Mounibe Diarra ${ }^{3}$, Claire Rodriguez-Lafrasse ${ }^{4}$ \\ ${ }^{1}$ Laboratory of Biophysics, Department of Biology and Functional Explorations, Thies University, Thies, Senegal \\ ${ }^{2}$ Diagnostic Laboratory, Children Hospital of Diamniadio, Dakar, Senegal \\ ${ }^{3}$ Laboratory of Pharmaceutical Physics, Pharmacy Department, University Cheikh Anta Diop of Dakar (UCAD), Dakar, Senegal \\ ${ }^{4}$ Molecular and Cellular Radiobiology Laboratory, University of Claude Bernard, Villeurbanne, France \\ ${ }^{5}$ Service of Hematology, National Centre of Blood Transfusion (CNTS), Department of Applied Biological and Pharmaceutical Sciences, \\ Faculty of Medicine, Pharmacy and Odontology (FMPO), University Cheikh Anta Diop of Dakar (UCAD), Dakar, Senegal \\ ${ }^{6}$ Research Institute in Oncology \& Haematology (RIOH), Cancer Care Manitoba (CCMB), Department of Physiology \& Pathophysiology, \\ University of Manitoba (UFM), Winnipeg, Manitoba, Canada \\ ${ }^{7}$ Cancer Institute, Department of surgery, University Cheikh Anta Diop of Dakar (UCAD), Dakar, Senegal
}

\section{Email address:}

soumboun@hotmail.com (M. Soumboundou),marie-therese.aloy@univ-lyon1.fr (M. T. Aloy), macoura.gadji@ucad.edu.sn (M. Gadji), celine.malesys@univ-lyon1.fr (C. Malesys),mbaygo@yahoo.fr (G. Mbaye),mounbeni@yahoo.fr (M. Diarra),

claire.lafrasse-rodriguez@univ-lyon1.fr (C. Rodriguez-Lafrasse)

*Corresponding author

\section{To cite this article:}

Mamadou Soumboundou, Marie Thérèse Aloy, Macoura Gadji, Celine Malesys, Gora Mbaye, Ahmadou Dem, Mounibe Diarra, Claire Rodriguez-Lafrasse. Determination of Endogenous Radioresistance Biomarkers on HeLa Cells. European Journal of Biophysics.

Vol. 8, No. 2, 2020, pp. 76-81. doi: 10.11648/j.ejb.20200802.18

Received: October 18, 2019; Accepted: November 27, 2019; Published: December 28, 2020

\begin{abstract}
The Genes such as carbonic anhydrase IX (CAIX), hypoxia inducible factor-1 (HIF-1) and vascular endothelial growth factor (VEGF) have been suggested as hypoxic biomarkers in cancer. Indeed, these endogenous biomarkers have been shown to have stronger prognostic value response after treatment by irradiation. However, inconsistent results suggest that factors other than oxygen influence their expression. This present study deciphers the level of expression of different radioresistance biomarkers in both normoxia and hypoxia conditions followed by irradiation of human ovarian tumor cell lines (uterine cervix squamous cell carcinoma (HeLa). HeLa cells were submitted to hypoxia $\left(1 \% \mathrm{O}_{2}\right)$ conditions in a Thermo Scientific Heracell i $\mathrm{CO}_{2}$ incubator. The cells were subjected to two doses 4-10 Gy irradiation and re-incubate in their starting conditions for 4 hours, then fixed in $4 \%$ paraformaldehyde for $20 \mathrm{~min}$. Protein expressions were assessed by immunocytochemistry staining and fluorescent images were captured by a Axio Imager Z1 fluorescence microscope with oil immersion lens at $63 \times$ magnification. In normoxia conditions there was no modification of the level of expression of the CAIX after irradiation. However, an increasing expression level of VEGF was noted. The level of expression of HIF-1 in normoxia was low compared to the other two proteins (CAIX and VEGF). Hypoxia conditions at 2\% resulted in a low expression of CAIX and VEGF before and after irradiation at $10 \mathrm{~Gy}$ in HeLa cells. HIF-1 had a maximum expression level compared to CAIX and VEGF at $2 \%$ oxygen after irradiation in HeLa cells. As tumor hypoxia occurs in a deprived microenvironment, other environmental factors such as irradiation might interact with the effect of low oxygen concentration on gene expression. This study shows that irradiation of HeLa cells has a profound influence on the oxygen dependent induction of certain endogenous hypoxic markers as HIF-1, CAIX, and VEGF.
\end{abstract}

Keywords: Radioresistance, Biomarkers, Hypoxia, Normoxia, DNA Repair HELA Cells 


\section{Introduction}

Cervical cancer is the third most commonly diagnosed cancer and the fourth leading cause of cancer death in women worldwide [1].

With over 528,000 new cases and more than 266,000 deaths in 2012 alone, cervical cancer is the fourth most common cancer in women worldwide, and second for women ages 15 to 44 [2]. Roughly 740 deaths per day occur due to cervical cancer, making it the second most common cause of cancer death in women [2].

Approximately $85 \%$ of the worldwide deaths from cervical cancer occur in underdeveloped or developing countries, and the death rate is 18 times higher in low income and middle income countries compared with wealthier countries [3].

Radiotherapy (RT) is the first treatment option offered to a majority of patient cases, since most of them present in clinical stages IIB, III or IV [4]. Clinical prognostic factors include tumor stage and nodal involvement, although metabolic response after treatment has been shown to have even stronger prognostic value [5].

A wide variation in the degree of normal tissue injury and tumor response have been observed in patients receiving identical RT. Considerable evidence has emerged that these wide variations in tumor response and normal tissue injury are largely caused by individual differences in intrinsic radiosensitivity [6].

A common feature of most tumors is a low level of oxygen, called hypoxia, the severity of which varies between tumor types [7].

In solid tumors, hypoxia has been proved to be a fundamental pathological feature that exerts numerous effects on the biological behavior of cancer cells. Because of the rapid growth of tumors, cancerous tissues grow beyond the physiological oxygen diffusion limit, and cancer cells activate angiogenesis, a process of generating new blood vessels stemming from the preexisting vasculature [8].

In many malignant tumors, hypoxia microenvironment is associated with poor prognosis, tumor aggressiveness and metastasis, post-treatment recurrence and resistance to radiation therapy [9].

To improve the outcome of cervical cancer, reliable biomarkers can identify patients with aggressive behavior and treatment of resistant tumors are needed [10].

Several endogenous proteins have been suggested as potential biomarkers of tumor hypoxia, including hypoxia-inducible factor- $1 \alpha$ (HIF-1 $\alpha$, the $\alpha$ subunit of the transcription factor HIF1) and downstream target genes of HIF-1 such as vascular endothelial growth factor-A (VEGF-A), glucose transporter-1, and carbonic anhydrase IX (CAIX) [11].

HIF- $1 \alpha$ overexpression is associated with an increased risk of mortality in early-stage of cervical carcinoma [12]. Among the target genes of HIF-1, CAIX seems to have the greatest potential as a biomarker of tumor hypoxia in cervical carcinoma. CAIX appears to be strictly regulated by hypoxia via HIF-1 $\alpha$ and shows strong up-regulation in hypoxic regions of tumors of the cervix [13].
Moreover, high level of CAIX in cervical tumors has been shown to be associated with lymph node metastasis and to predict poor overall and metastasis-free survival rates after radiation therapy [14].

An era of personalized cancer medicine in which biomarkers can be used to tailor treatment to each specific patient is a major goal in oncology. Prognostic biomarkers provide information regarding disease outcome regardless of the treatment received and predictive biomarkers determine which patients will probably derive benefit from a specific therapy [15].

The aim of this study was to evaluate the radiobiological response and the level of expression of endogenous radioresistance biomarkers on cervical cancer cell line (HeLa cells using both normoxia and hypoxia conditions followed by irradiation. These results will allow us to search these endogenous radioresistance biomarkers in a series of Senegalese women with cervix cancers attending radiotherapy.

\section{Materials and Experiments}

\subsection{Cell Culture}

The cervical cancer HeLa cell line was purchased from the Cell Bank of Laboratory of Cellular and Molecular Radiobiology, EMR3738, Faculty of Medicine Lyon-South (Lyon, France). After thawing, the HeLa cells were cultured in Dulbecco's modified Eagle's medium (DMEM) and Glutamax-I (4, $5 \mathrm{~g} / \mathrm{L}$ D-Glucose) supplemented with $10 \%$ fetal bovine serum, $100 \mathrm{U} / \mathrm{ml}$ penicillin, $100 \mathrm{ug} / \mathrm{ml}$ streptomycin and then incubated at $37^{\circ} \mathrm{C}$ in a humidified atmosphere containing $5 \%$ $\mathrm{CO}_{2}$ incubator (KM-CC17R2, JAPAN). To detach HeLa cells in the logarithmic growth phase, flasks were trypsinized with 1, 5 $\mathrm{ml}$ trypsin (Gibco, Thermo Fisher-US, Waltham, Massachusetts) followed by cell counting.

\subsection{Reagents and Antibodies}

The HIF-1 $\alpha$ antibody (sc-53546), the VEGF mouse monoclonal antibody (sc-7269) and CAIX rabbit polyclonal antibody (sc-25599) were purchased from Santa Cruz Biotechnology, INC 1: 200 (Santa Cruz Biotechnology, Dallas, US).

\subsection{Irradiation Technique}

After 24 hours of incubation at $37^{\circ} \mathrm{C}$, allowing sufficient time for cells to completely adhere to the surface, the cells were treated with radiation in megavoltage radiotherapy machines. Different dose ranging from low dose region (1 Gy to $2 \mathrm{~Gy}$ ) and beyond, up to $4 \mathrm{~Gy}$ were used (6 Gy to $8 \mathrm{~Gy}$ ). $\mathrm{X}$-ray irradiation was performed at $250 \mathrm{kV}$ delivered at a dose rate of $2 \mathrm{~Gy} / \mathrm{min}$ using a X-Rad 320 irradiator (Precision X-ray Inc., North Branford, CT).

\subsection{Clonogenic Cell Death Assays}

Cell survival curves were generated using a standard 
colony formation assay. HeLa cells were seeded in $25 \mathrm{~cm}^{2}$ culture flasks at densities of $100-1600$ cells $/ \mathrm{cm}^{2}$ to yield 10-200 colonies after irradiation with doses of $1,2,4,6,8$ Gy. After 6 days at $37^{\circ} \mathrm{C}$ corresponding to six cellular dividing, cells were washed with phosphate buffered saline (PBS), fixed with methanol and stained with Giemsa (Sigma-Aldrich Saint-Louis, Missouri, US). Viable colonies (colonies with cells more than 50) were counted on a Colcount ${ }^{\mathrm{TM}}$ (OXFORD Optronix; Nottingham, U.K). In non-irradiated plates (control) percentages of cells seeded that form viable colonies provided plating efficiency (PE). Surviving fraction was calculated using the following formula (1) [16].

$$
\frac{\text { Colonies counted }}{\text { Colonies seeded }} \times(\text { P.E/100) }
$$

Clonogenic survival curves were fitted according to the linear quadratic equation (2); where SF is the surviving fraction, $\alpha$ and $\beta$ represent the initial slope (probability of lethal events) and the terminal slope (sublethal events) constants respectively; and D represents the irradiation dose. The SF at 2 Gy (SF2) was used as an index of radiosensitivity.

$$
S F 2=e^{-\left[\alpha D+\beta D^{2}\right]}
$$

\section{5. $\gamma-H 2 A X$ Immunofluorescence Assay}

Phospho-H2AX foci immunofluorescence was detected to monitor the DNA DSBs and DSB repair capacity of HeLa cells. The detection of $\gamma-\mathrm{H} 2 \mathrm{AX}$ foci was assayed by immunocytochemistry. Briefly, cells were fixed in $4 \%$ paraformaldehyde for $20 \mathrm{~min}$, and immunodetection was performed as described by Hanot et al [17]. Foci were visualized on an Axio Imager Z1 fluorescence microscope (Carl Zeiss S. A. S., Le Pecq, France) and $\gamma$-H2AX staining was quantified using Image $J$ software. The results were expressed as mean \pm SD of a minimal counting of 100 nuclei per slide performed in duplicate.

\subsection{Endogenous Markers of Hypoxia Induction}

Cells $\left(2 \times 10^{5}\right)$ were seeded in six-well plates and cultured until they reached $30 \%$ confluence. Cells were additionally cultured in two different conditions for $24 \mathrm{~h}$. In normoxia conditions (control group), cells were incubated at $37^{\circ} \mathrm{C}$ in a humidified atmosphere containing $5 \% \mathrm{CO}_{2}$ incubator (KMCC17R2, JAPAN); and in hypoxia conditions (hypoxia group) cells were generated in a Thermo Scientific Heracell $\mathrm{i}$ $\mathrm{CO}_{2}$ incubator.

The cells were subjected to two doses 4-10 Gy irradiation and re-incubate in their starting conditions for 4 hours fixed in $4 \%$ paraformaldehyde for $20 \mathrm{~min}$.

\subsection{Immunocytochemistry Staining}

Cells cultured on $15 \mathrm{~mm}$ cover slips were washed twice in PBS $1 x$ and fixed with $4 \%$ paraformaldehyde at $4{ }^{\circ} \mathrm{C}$ for $30 \mathrm{~min}$. After fixation, the cells were permeabilized using $0.1 \%$ Triton $\mathrm{X}-100$ for $10 \mathrm{~min}$ and blocked with $5 \%$ bovine serum albumin for $30 \mathrm{~min}$ at room temperature. The samples were then incubated with primary antibodies at $4{ }^{\circ} \mathrm{C}$ overnight, followed by incubation with Alexa Fluor 488-conjugated goat anti-rabbit secondary antibody (Thermo Fisher Scientific, USA) for $1 \mathrm{~h}$ at room temperature. The cover slips were then fixed on slides using Fluor shield Mounting Medium with DAPI (Abcam, USA), which contains DAPI for the counterstaining of nuclei. Fluorescent images were captured by an Axio Imager Z1 fluorescence microscope (Carl Zeiss S. A. S., Le Pecq, France) with oil immersion lens at $63 \times$ magnification.

\section{Results}

\subsection{Clonogenic Cell Death Assays}

The results concerning clonogenic cell survival in HeLa cells following X-ray irradiations at $250 \mathrm{KeV}$ are shown in Figure 1. Thus, the survival factor at 2 Gy after exposure to $250 \mathrm{KeV}$ X-ray exposures was 0.48 for HeLa cells. This result shows that HeLa cells are resistant to X-ray irradiation (Figure 1).

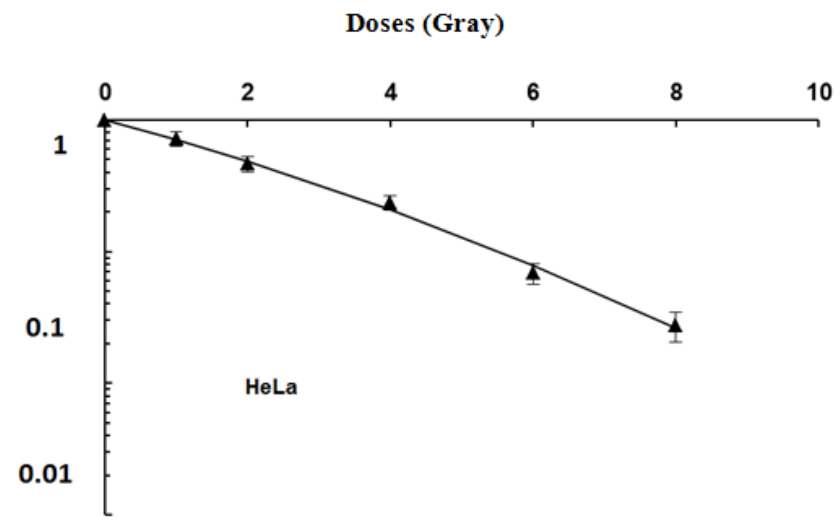

$\mathrm{SF} 2=0.48$

Figure 1. Dose-response curves for cell killing following X-ray exposures. The survival curve was fitted according to linear quadratic equation. Values represent the mean $\pm S D$ of three independent experiments.

\subsection{Quantification of $\gamma-H 2 A X$ Foci}

To confirm the radioresistant character of the HeLa cells, we determined the residual double strand breaks by quantifying the initial (30 min) and residual (24 h) doublestrand breaks (DSB) as shown by $\gamma$-H2AX foci (Figure 2). HeLa cells show $2.3 \pm 0.2$ foci / cell at $30 \mathrm{~min}$; exposure to a 2 Gy irradiation induced the formation of $17.9 \pm 0.1$ foci/cell. As expected $24 \mathrm{~h}$ after irradiation, DSBs returned to basal levels given the radioresistance of HeLa cells (Figure 2). 


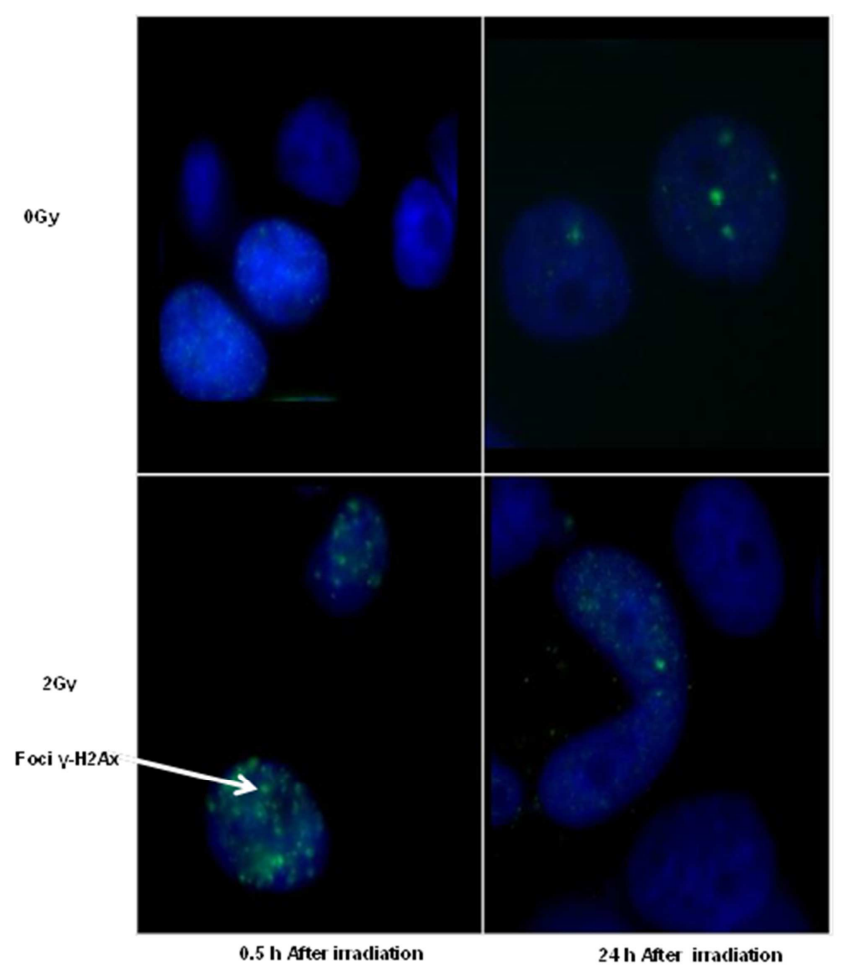

Figure 2. Quantification of $\gamma-H 2 A X$ foci 0.5 and 24 h after treatment of HeLa cells with 2 Gy irradiation.

\subsection{Immunocytochemistry}

Qualitative immunocytochemistry analysis showed increased expression of HIF-1 $\alpha$, CAIX and VEGF at normoxia $24 \mathrm{~h}$ after X-ray irradiation at 10 Gy compared with none irradiated cells (Figure 3). However, the expression of CAIX was more visible compared to VEGF and HIF-1 $\alpha$ in normoxia conditions (Figure 4).

Normoxia 4 h after irradiation 10 Gy

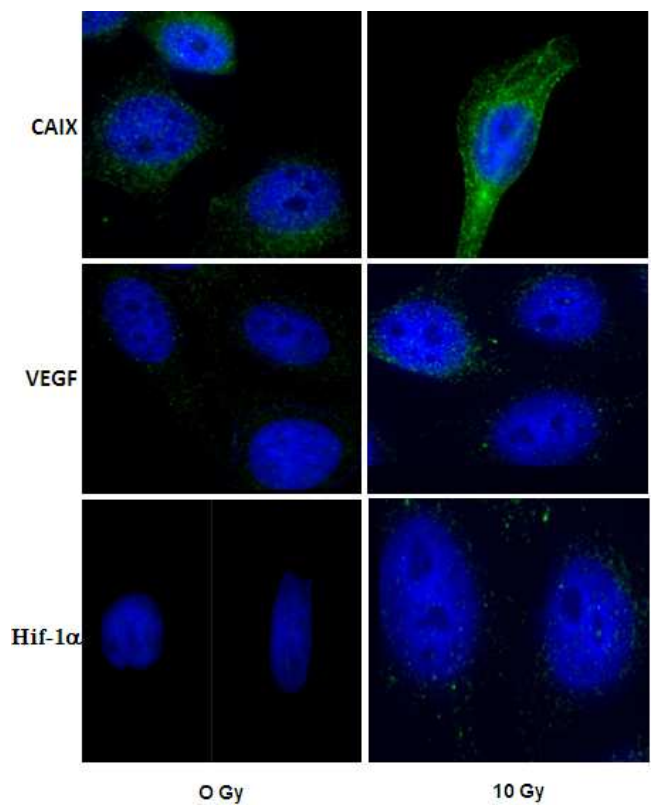

Figure 3. Photograph of an immunofluorescence carried out on a HeLa cells, treated with an anti-Hif-1 $\alpha$, anti-vegf and CAIX antibody (green proteins) and by the nuclear dye DAPI (blue).
We studied the effect of hypoxia on the level of expression of biomarkers related to its induction in order to answer to this question of whether the level of expression of the different markers could be a function of a physiological condition, namely tumor hypoxia.

Thus, an overexpression of HIF- $1 \alpha$ was noted when the cells were maintained in hypoxia $\left(24 \mathrm{~h}\right.$ in hypoxia $1 \% \mathrm{O}_{2}$, Irradiation $10 \mathrm{~Gy}, 4 \mathrm{~h}$ in Hypoxia $1 \% \mathrm{O}_{2}$ ). However, the level of expressions of CAIX and VEGF was not microscopically significant compared to expression in normoxia conditions (Figure 3).

- $24 \mathrm{~h}$ in hypoxia $1 \% \mathrm{O}_{2}$, irradiation 1

CAIX

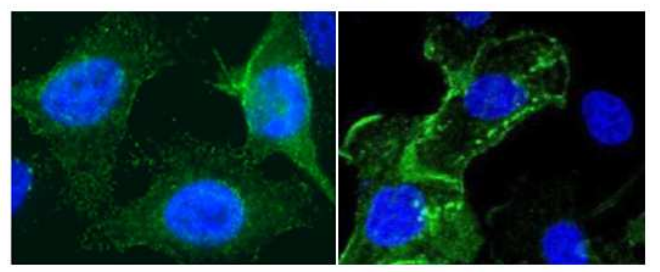

VEGF

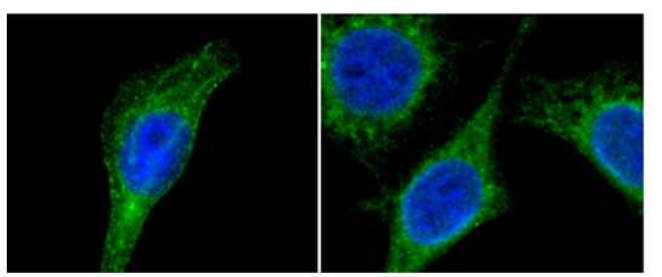

HIF-1 $\alpha$
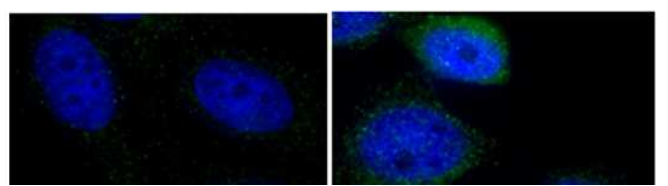

Figure 4. Photograph of an immunofluorescence carried out on a HeLa cells, treated with an anti-Hif-1 $\alpha$, anti-vegf and CAIX antibody (green proteins) and by the nuclear dye DAPI (blue).

\section{Discussion}

Tumor hypoxia represents a significant obstacle in the clinical management of locally advanced carcinoma of the uterine cervix, by increasing malignancy and inducing treatment resistance [11].

A major player in the hypoxia response is HIF-1 $\alpha$, with its downstream target genes involved in cell survival, metabolism, angiogenesis, and metastasis [18].

Based on a number of clinical studies, several investigators have promoted the view that the expression of HIF-1 $\alpha$ and its target genes may be useful biomarkers of tumor hypoxia, aggressiveness, and radiation resistance in cervical carcinoma [19]. In this present preclinical study, we challenged this view by searching correlations between tumor hypoxia and the expression of HIF-1 $\alpha$, VEGF and CAIX in HeLa cervical carcinoma cells lines.

A significant advantage of preclinical investigations over clinical investigations is the measurement conditions can be kept unchanged during the clinical study. A major disadvantage of preclinical studies is experimental cells may not be necessary valid models of human cancer. 
Radiotherapy is still one of the best cervical cancer therapy. Radiotherapy technology has evolved remarkably during the past decade, and radiation can be precisely delivered, thereby allowing higher doses to the tumor and reducing doses to surrounding normal tissues [20].

Tumor recurrence following a course of radiation therapy results from radiation resistance in some cells which can survive and re-proliferate. One of the major reasons for the failure of radiation therapy is hypoxia [21, 22].

It is well known that HIF-1 $\alpha$ promotes tumor radioresistance through stimulation of endothelial cell survival [23]. HIF-1 $\alpha$ expression is associated with a reduced survival in a variety of human cancers including uterine cervix [24], ovary [25], esophagus, lung, and breast and may also influence resistance to therapy in several cancer types [26].

Potential explanations for these differences include tumors heterogeneity in the level of oxygenation and hence HIF$1 \alpha$ expression; and HIF- $1 \alpha$ is rapidly degradated with restoration of normoxia, making HIF-1 $\alpha$ more reflective of acute rather than chronic tumor hypoxia [27].

Of all the considered biomarkers in this preclinical study, HIF-1 $\alpha$, VEGF, CAIX proteins showed significant findings in hypoxia after irradiation but not in normoxia. Indeed, in normoxia conditions the low expression of HIF-1 $\alpha$ found can be explained by the fact HIF- $1 \alpha$ 's rapid degradation with restoration of normoxia, making HIF-1 $\alpha$ more reflective of acute rather than chronic tumor hypoxia [27].

That is why; the data suggest that for each cancer it will be important to determine whether HIF-1 $\alpha$ overexpression, either alone or in combination with other markers such as p53 or BCL2, can identify a subset of patients with more aggressive disease for whom standard therapy will not be sufficient to ensure a high probability of survival.

Additionally, the transiently expression of HIF-1 $\alpha$ compared with metabolic proteins expression, resulting in increased potential false-negative results from HIF- $1 \alpha$ staining.

Our results have shown that CAIX and VEGF are overexpressed on radioresistant $\mathrm{HeLa}$ cells line in both conditions $63 \times$ magnification. normoxia and hypoxia.

In fact, several studies have demonstrated that high CAIX and VEGF expression level in tumor cells correlate with greater malignant potential, more aggressive growth characteristics and risk of metastasis [28, 29] $63 \times$ magnification [30].

This result compared to the level of expression of the CAIX proves its usefulness in medical imaging because it is not depressed by the physiological conditions. Pastorekova et al., 2008 had already mentioned the usefulness of CAIX as an emerging target for cancer therapy and functional imaging [31], furthermore broadening the potential clinical value of the CAIX biomarker in the detection, treatment and management of women with cervical cancer.

\section{Conclusion}

In radiotherapy, the treatment is adapted to each individual to protect healthy tissues but delivers most of time a standard dose according to the tumor histology and site. The only biomarkers studied to individualize the treatment are the HPV status with radiation dose de-escalation strategies, and tumor hypoxia with dose escalation to hypoxic subvolumes using FMISO- or FAZA-PET imaging.

These preclinical results on the radio-resistant HeLa cells show different level of expression of radioresistant proteins such as HIF-1 $\alpha$, CAIX and VEGF. Thus, the development of prognostic index according to the different biomarkers is feasible by immunohistochemistry on human cervical cancer biopsies. These different biomarkers would allow better selection and follow-up of patients bearing cervical cancer, and an integration of the intrinsic biological data of the tumor in the treatment.

Finally, the systematic search for potential biomarkers predicting the response to irradiation could be used to modulate therapy in the event of a positive response biomarker, or, conversely, to stop, as part of a treatment associated with the treatment irradiation.

\section{Acknowledgements}

MS hold a PhD scholarship from French cooperation "Cooperation Française".

\section{Conflict of Interest}

The authors have no conflict of interest to disclose.

\section{References}

[1] Bray F, Ferlay J, Soerjomataram I, Siegel R L and Jemal A. Global cancer statistics 2018: GLOBOCAN estimates of incidence and mortality worldwide for 36 cancers in 185 countries. CA Cancer J Clin. 68, 6, 2018, pp 394-424.

[2] William S; Monica A. B; Amishi B; Linus T. C and Gaffney D K. Cervical Cancer: A Global Health Crisis. Cancer, 123, 13, 2017, pp 2404-2412.

[3] World Health Organization. Human papillomavirus (HPV) and cervical cancer. http://www.who.int/mediacentre/factsheets/fs380/en/. Accessed September 05, 2019.

[4] Mukherjee G, Freeman A, Moore R, Kumaraswamy and Stanley MA. Biologic factors and response to radiotherapy in carcinoma of the cervix. Int J Gynecol Cancer. 11, 3, 2001, pp 187-193.

[5] Schwarz JK, Siegel BA, Dehdashti F and Grigsby PW. Metabolic response on post-therapy FDG-PET predicts patterns of failure after radiotherapy for cervical cancer. Int $J$ Radiat Oncol Biol Phys. 83, 2012, pp 185-190.

[6] Pawlik TM and Keyomarsi K. Role of cell cycle in mediating sensitivity to radiotherapy. Int J Radiat Oncol Biol Phys. 59, 4, 2004, pp 928-942.

[7] Barbara M, Pilar de la P, Feda A and Abdel K A. The role of hypoxia in cancer progression, angiogenesis, metastasis, and resistance to therapy. Hypoxia (Auckl). 3, 2015, pp 83-92. 
[8] Ribatti D and Crivellato E. "Sprouting angiogenesis", a reappraisal. Dev Biol., 372, 2, 2012, pp 157-165.

[9] Bhaskar A and Tiwary B. N. Hypoxia inducible factor-1 alpha and multiple myeloma. Int. J. Adv. Res. 4, 1, 2016, pp 706715 .

[10] Klopp AH and Eifel PJ. Biological predictors of cervical cancer response to radiation therapy. Semin Radiat Oncol. 22, 2, 2012, pp 143-150.

[11] Christine Ellingsen, Lise Mari K. Andersen, Kanthi Galappathi and Einar K. Rofstad. Hypoxia biomarkers in squamous cell carcinoma of the uterine cervix. BMC Cancer. 15, 2015: 805 .

[12] Birner P, Schindl M, Obermair A, Plank C and Oberhuber G. Overexpression of hypoxia-inducible factor $1 \alpha$ is a marker for an unfavorable prognosis in early-stage invasive cervical cancer. Cancer Res. 60, 17, 2000, pp 4693-4596.

[13] Kaluz S, Kaluzová M, Liao SY, Lerman M and Stanbridge EJ. Transcriptional control of the tumor- and hypoxia-marker carbonic anhydrase 9: a one transcription factor (HIF-1) show? Biochim Biophys Acta. 1795, 2, 2009, pp 162-172.

[14] Loncaster JA, Harris AL, Davidson SE, Logue JP and Wycoff CC. Carbonic anhydrase (CA IX) expression, a potential new intrinsic marker of hypoxia: correlations with tumor oxygen measurements and prognosis in locally advanced carcinoma of the cervix. Cancer Res. 61, 17, 2001, pp 6394-6399.

[15] Forker L. J, Choudhury A and Kiltie A. E. Biomarkers of Tumour Radiosensitivity and Predicting Benefit from Radiotherapy. Clinical Oncology. 27, 10, 2015, pp 561-569.

[16] Franken NAP, Rodermond HM, Stap J, Haveman J and Van Bree C. Clonogenic assay of cells in vitro. Nat Protocols. 1, 5, 2006, pp 2315-2319.

[17] Hanot M, Boivin A, Malesys C, Beuve M and RodriguezLafrasse C. Glutathione depletion and carbon ion radiation potentiates clustered DNA lesions, cell death and prevents chromosomal changes in cancer cells progeny. Plos One 2012; $7,11,2012, e 44367$.

[18] Dellas K, Bache M, Pigorsch SU, Taubert H and Haensgen G. Prognostic impact of HIF-1 $\alpha$ expression in patients with definitive radiotherapy for cervical cancer. Strahlenther Onkol. 184, 3, 2008, pp 169-174.

[19] Hasmim M, Messai Y, Noman M. Z and Chouaib S. L'hypoxie tumorale: un déterminant clé de la réactivité stromale et de la réponse antitumorale. Med Sci (Paris). 2014, 30 (4), p 422428 .
[20] Jinsil S. Challenge and hope in radiotherapy of hepatocellular carcinoma. Yonsei Med J. 50, 5, 2009, pp 601-612.

[21] Moeller BJ and Dewhirst MW. HIF-1 and tumour radiosensitivity. Br J Cancer. 95, 1, 2006, pp 1-5.

[22] Bertout JA, Patel SA and Simon MC. The impact of $\mathrm{O}_{2}$ availability on human cancer. Nat Rev Cancer. 8, 12, 2008, pp 967-975.

[23] Moeller BJ, Dreher MR, Rabbani ZN, Schroeder T and Dewhirst MW. Pleiotropic effects of HIF-1 blockade on tumor radiosensitivity. Cancer Cell. 8, 2, 2005, pp 99-110.

[24] Burri P, Djonov V, Aebersold DM, Lindel K and Gruber G. Significant correlation of hypoxia-inducible factor- $1 \alpha$ with treatment outcome in cervical cancer treated with radical radiotherapy. Int J Radiat Oncol Biol Phys. 56, 2, 2003, pp 494-501.

[25] Birner P, Schindl M, Obermair A, Breitenecker G and Oberhuber G. Expression of hypoxia-inducible factor $1 \alpha$ in epithelial ovarian tumors: its impact on prognosis and on response to chemotherapy. Clin Cancer Res. 7, 6, 2001, pp $1661-1668$

[26] Daniele G, Alfredo B, Maria P. B, Leticia C and Stephen B. Fox. Hypoxia-Inducible Factor-1 $\alpha$ Expression Predicts a Poor Response to Primary Chemoendocrine Therapy and DiseaseFree Survival in Primary Human Breast Cancer. Clin Cancer Res. 12, 15, 2006, pp 4562-4568.

[27] Hutchison GJ, Valentine HR, Loncaster JA, Davidson SE and West CM. Hypoxia-inducible factor 1alpha expression as an intrinsic marker of hypoxia: correlation with tumor oxygen, pimonidazole measurements, and outcome in locally advanced carcinoma of the cervix. Clin Cancer Res. 10, 24, 2004, pp $8405-8412$.

[28] Yuefen Z, Chao C, Motoko O-M, Yun K and Tomio I. The suppression of hypoxia inducible factor and vascular endothelial growth factor by siRNA does not affect the radiation sensitivity of multicellular tumor spheroids. $J$ Radiat Res, 51, 2010, pp 47-55.

[29] Semenza GL. Targeting HIF-1 for cancer therapy. Nat Rev Cancer. 3, 10, 2003, pp 721-732.

[30] Lee S, Shin HJ, Han IO, Hong EK and Kim JY. Tumor carbonic anhydrase 9 expression is associated with the presence of lymph node metastases in uterine cervical cancer. Cancer Sci, 98, 3, 2007, p 329-333.

[31] Pastorekova S, Zatovicova $\mathrm{M}$ and Pastorek J. Cancerassociated carbonic anhydrases and their inhibition. Curr Pharm Des. 14, 7, 2008, pp 685-698. 